\title{
AN INTERCROSS FAMILY WITH AN UNUSUAL PROPERTY
}

\author{
K. MATHER and M. J. LAWRENCE \\ Department of Genetics, University of Birmingham, Birmingham B/5 2TT
}

Received 8.ii.79

\section{SUMMARY}

The detection and estimation of linkage is described for a completely classified intercross from which one array is missing. Provided that the missing array involves a gamete of non-recombinant type, this kind of family is capable of yielding more information about the linkage value in the affected parent than a completely represented family.

\section{INTRODUCTION}

Lawrence, Cornish and Hayward (1979) have shown that it is possible to obtain independent estimates of male and female recombination frequency from a completely classified intercross family in which three or four alleles are segregating at each of a pair of linked loci. In the present note we consider a variant of this design with a rather unexpected property. It is convenient to refer to the first intercross as a type 1 family and to the one to be considered here as a type 1A family.

Consider a cross between an individual of genotype $A_{1} B_{1} / A_{2} B_{2}$ used as female and another of genotype $A_{1} B_{1} / A_{3} B_{3}$ used as male, where $A_{1}, A_{2}$ and $A_{3}$ are alleles at the first and $B_{1}, B_{2}$ and $B_{3}$ are those at the second locus. Suppose that male gametes of genotype $A_{1} B_{1}$ either do not occur, because of complementary gametic lethality, or fail to function, because

\section{TABLE 1}

The expected composition of progeny produced by crossing an $\mathrm{A}_{1} \mathrm{~B}_{1} / \mathrm{A}_{2} \mathrm{~B}_{2}$ individual used as female with an $\mathrm{A}_{1} \mathrm{~B}_{1} / \mathrm{A}_{3} \mathrm{~B}_{3}$ individual used as a male parent when male gametes of genotype $\mathrm{A}_{1} \mathrm{~B}_{1}$ fail to survive or function. The $\mathrm{n}_{1 \mathrm{j}}$ 's are the number observed in each class and $\mathrm{t}_{\mathrm{m}}=\left(1+\mathrm{p}_{\mathrm{m}}\right)$. Because of the missing male array, the row totals are partial sums. The grand total, $\mathrm{n} .$. is written as $\mathrm{n}$ in the text

\begin{tabular}{|c|c|c|c|c|c|}
\hline & $\begin{array}{c}\left(A_{1} B_{1}\right) \\
(0)\end{array}$ & $\begin{array}{c}A_{1} B_{3} \\
p_{m} / t_{m}\end{array}$ & $\begin{array}{c}A_{3} B_{1} \\
p_{m} / t_{m}\end{array}$ & $\begin{array}{l}A_{3} B_{3} \\
q_{m} / t_{m}\end{array}$ & $\begin{array}{l}\text { Row } \\
\text { totals }\end{array}$ \\
\hline $\begin{array}{c}A_{1} B_{1} \\
q_{f} / 2\end{array}$ & - & $\begin{array}{c}A_{1} B_{1} / A_{1} B_{3} \\
q_{f} p_{m} / 2 t_{m} \\
n_{12}\end{array}$ & $\begin{array}{c}A_{1} B_{1} / A_{3} B_{1} \\
q_{f} p_{m} / 2 t_{m} \\
n_{13}\end{array}$ & $\begin{array}{c}A_{1} B_{1} / A_{3} B_{3} \\
q_{f} q_{m} / 2 t_{m} \\
n_{14}\end{array}$ & $\begin{array}{r}q_{f} / 2 \\
n_{1} .\end{array}$ \\
\hline $\begin{array}{l}A_{1} B_{2} \\
p_{f} / 2\end{array}$ & - & $\begin{array}{c}A_{1} B_{2} / A_{1} B_{3} \\
p_{f} p_{m} / 2 t_{m} \\
n_{22}\end{array}$ & $\begin{array}{c}A_{1} B_{2} / A_{3} B_{1} \\
p_{f} p_{m} / 2 t_{m} \\
n_{23}\end{array}$ & $\begin{array}{c}A_{1} B_{2} / A_{3} B_{3} \\
p_{f} q_{m} / 2 t_{m} \\
n_{24}\end{array}$ & $\begin{array}{c}p_{f} / 2 \\
n_{2} .\end{array}$ \\
\hline $\begin{array}{c}A_{2} B_{1} \\
p_{f} / 2\end{array}$ & - & $\begin{array}{c}A_{2} B_{1} / A_{1} B_{3} \\
p_{f} p_{m} / 2 t_{m} \\
n_{32}\end{array}$ & $\begin{array}{c}A_{2} B_{1} / A_{3} B_{1} \\
p_{f} p_{m} / 2 t_{m} \\
n_{33}\end{array}$ & $\begin{array}{c}A_{2} B_{1} / A_{3} B_{3} \\
p_{f} q_{m} / 2 t_{m} \\
n_{34}\end{array}$ & $\begin{array}{c}p_{f} / 2 \\
n_{3} .\end{array}$ \\
\hline $\begin{array}{c}A_{2} B_{2} \\
q_{f} / 2\end{array}$ & - & $\begin{array}{c}A_{2} B_{2} / A_{1} B_{3} \\
q_{f} p_{m} / 2 t_{m} \\
n_{42}\end{array}$ & $\begin{array}{c}A_{2} B_{2} / A_{3} B_{1} \\
q_{f} p_{m} / 2 t_{m} \\
n_{43}\end{array}$ & $\begin{array}{c}A_{2} B_{2} / A_{3} B_{3} \\
q_{f} q_{m} / 2 t_{m} \\
n_{44}\end{array}$ & $\begin{array}{l}q_{f} / 2 \\
n_{4} .\end{array}$ \\
\hline Column totals & - & $\begin{array}{r}p_{m} / t_{m} \\
n_{.2}\end{array}$ & $\begin{array}{r}p_{m} / t_{m} \\
n_{.3}\end{array}$ & $\begin{array}{c}q_{m} / t_{m} \\
n_{.4}\end{array}$ & $\begin{array}{c}1 \\
n . .\end{array}$ \\
\hline
\end{tabular}


of their incompatibility on stigmata which carry the same alleles. Then if $p_{f}$ is the frequency of recombination on the female and $p_{m}$ is the corresponding frequency on the male side of the cross and there are no further viability disturbances, the frequencies of the genotypes expected in the progeny are as shown in table $1\left(q_{f}=1-p_{f}\right.$ and $\left.q_{m}=1-p_{m}\right)$. The actual or effective absence on the male side of one of the four gametic genotypes means, of course, that the frequencies of the surviving three have to be divided by $t_{m}=\left(1+p_{m}\right)$ in order that they shall sum to unity; and that we now have 12 zygotic genotypes, rather than the 16 of the type 1 intercross in which all four kinds of male gamete participate in fertilisation. However, as in the latter, if there is no dominance at either locus, each genotype will produce a unique phenotype. The present family is thus an incompletely represented but completely classified intercross.

\section{Estimation of male AND female Linkage}

The test for linkage on the female side is the same as that for the type 1 family, namely:

$$
\begin{aligned}
\chi_{(1)}^{2} & =\left(n_{1 .}-n_{2 .}-n_{3 .}+n_{4 .}\right)^{2} / n \\
& =F^{2} / n
\end{aligned}
$$

where $F$ is written for $\left(n_{1},-n_{2 .}-n_{3 .}+n_{4}\right.$. $)$ and $n$ is written for the $n_{\text {.. }}$ of table 1. On the male side, however, the test for linkage now becomes:

$$
\chi_{(1)}^{2}=\left(2 n_{.4}-n_{.2}-n_{.3}\right)^{2} / 2 n=M^{2} / 2 n
$$

where $M$ is written for $\left(2 n_{.4}-n_{.2}-n_{.3}\right)$.

The joint test for linkage and the test of heterogeneity between the male and female sides will be discussed after the estimation of the linkage values has been considered.

Turning to the estimation of the linkage values, $p_{f}$ and $p_{m}$, for the female and male recombination fractions respectively, the logarithm of the likelihood is

$$
\begin{aligned}
L & =\left[\left(n_{22}+n_{23}+n_{24}\right)+\left(n_{32}+n_{33}+n_{34}\right)\right] \log p_{f} \\
& +\left[\left(n_{12}+n_{13}+n_{14}\right)+\left(n_{42}+n_{43}+n_{44}\right)\right] \log q_{f} \\
& +\left[\left(n_{12}+n_{22}+n_{32}+n_{42}\right)+\left(n_{13}+n_{23}+n_{33}+n_{34}\right)\right] \log p_{m} \\
& +\left[\left(n_{14}+n_{24}+n_{34}+n_{44}\right)\right] \log q_{m} \\
& -n \log \left(1+p_{m}\right)+\text { constants }
\end{aligned}
$$

which on partial differentiation in respect of $p_{f}$ and $p_{m}$ gives as the two equations of estimation:

$$
\begin{aligned}
& \frac{\partial L}{\partial p_{f}}=\frac{n_{2 .}+n_{3} .}{p_{f}}-\frac{n_{1 .}+n_{4 .}}{q_{f}}=0 \\
& \frac{\partial L}{\partial p_{m}}=\frac{n_{.2}+n_{.3}}{p_{m}}-\frac{n_{.4}}{q_{m}}-\frac{n}{1+p_{m}}=0
\end{aligned}
$$

The two estimates are thus

$$
p_{f}=\frac{n_{2 .}+n_{3} .}{n} \quad \text { and } \quad p_{m}=\frac{n_{.2}+n_{.3}}{n_{.4}+n}
$$


We note that on the female side both the equation of estimation and the estimate are the same as those from the type 1 intercross, except that the row sums involve three rather than four cells. Both the equation of estimation and the estimate of recombination frequency on the male side, on the other hand, are different from the previous case. Despite this, however, the estimates of $p_{f}$ and $p_{m}$ are still independent: indeed the log likelihood is composed of two completely separable parts, one dependent solely on $p_{f}$ and yielding the estimate of this parameter and the other similarly dependent solely on $p_{m}$.

The amounts of information about the estimates yielded by a family of the present type are:

$$
I_{p_{f}}=\frac{n}{p_{f} q_{f}} \quad \text { and } \quad I_{p_{m}}=\frac{2 n}{\left(1+p_{m}\right)^{2} p_{m} q_{m}}
$$

Again, as with the estimate itself, the amount of information about recombination on the female side of the cross is the same as that for the previous design. The corresponding statistics on the male side, however, take a novel form.

\section{The efficiency of the male estimate}

Now in the completely classified cross with 16 classes, the amount of information yielded by an individual in respect of $p_{m}$ is

$$
i_{p_{m}}=\frac{I_{p_{m}}}{n}=\frac{1}{p_{m} q_{m}}
$$

In the present case this quantity is

$$
i_{p_{m}}^{\prime}=\frac{I_{p_{m}}^{\prime}}{n}=\frac{2}{\left(1+p_{m}\right)^{2} p_{m} q_{m}}
$$

The relative efficiency of the type $1 \mathrm{~A}$ compared with the type 1 family in respect of the estimation of recombination on the male side is thus:

$$
\text { Efficiency } \%=i_{p_{m}}^{\prime} / i_{p_{m}}=\frac{2}{\left(1+p_{m}\right)^{2}} \times 100
$$

Since this efficiency depends on $p_{m}$, it is convenient to plot values of the former against the latter in the form of a graph (figure 1).

It is quite clear that for values of $p_{m}$ less than $\sqrt{ } 2-1=0.41$, the present design is more efficient than the previous one. Indeed with very tight linkage, this incomplete cross is nearly twice as efficient as the type 1 cross. This is, of course, a rather unexpected outcome since in general incomplete families are less informative than complete ones. However, it is worth emphasising that this gain in information is obtained only if the appropriate male array is missing. Thus in the present case we have assumed for the purpose of illustration that the linkage between $A_{1}$ and $B_{1}$ is in the coupling phase and that male gametes of genotype $A_{1} B_{1}$ fail to function. Gametes of this genotype are, of course, non-recombinant with respect to the alleles carried by the male parent. If this assumption about the phase of linkage is 
invalid, the present design is less efficient than the previous one. Thus if linkage is in the repulsion phase $(p>0.5$ in the graph), the efficiency of the type $1 \mathrm{~A}$ design can fall to no more than 50 per cent of that of the type 1 design. In practice, it may be possible to circumvent this difficulty by rearranging the cross so as to ensure that the missing array concerns a male

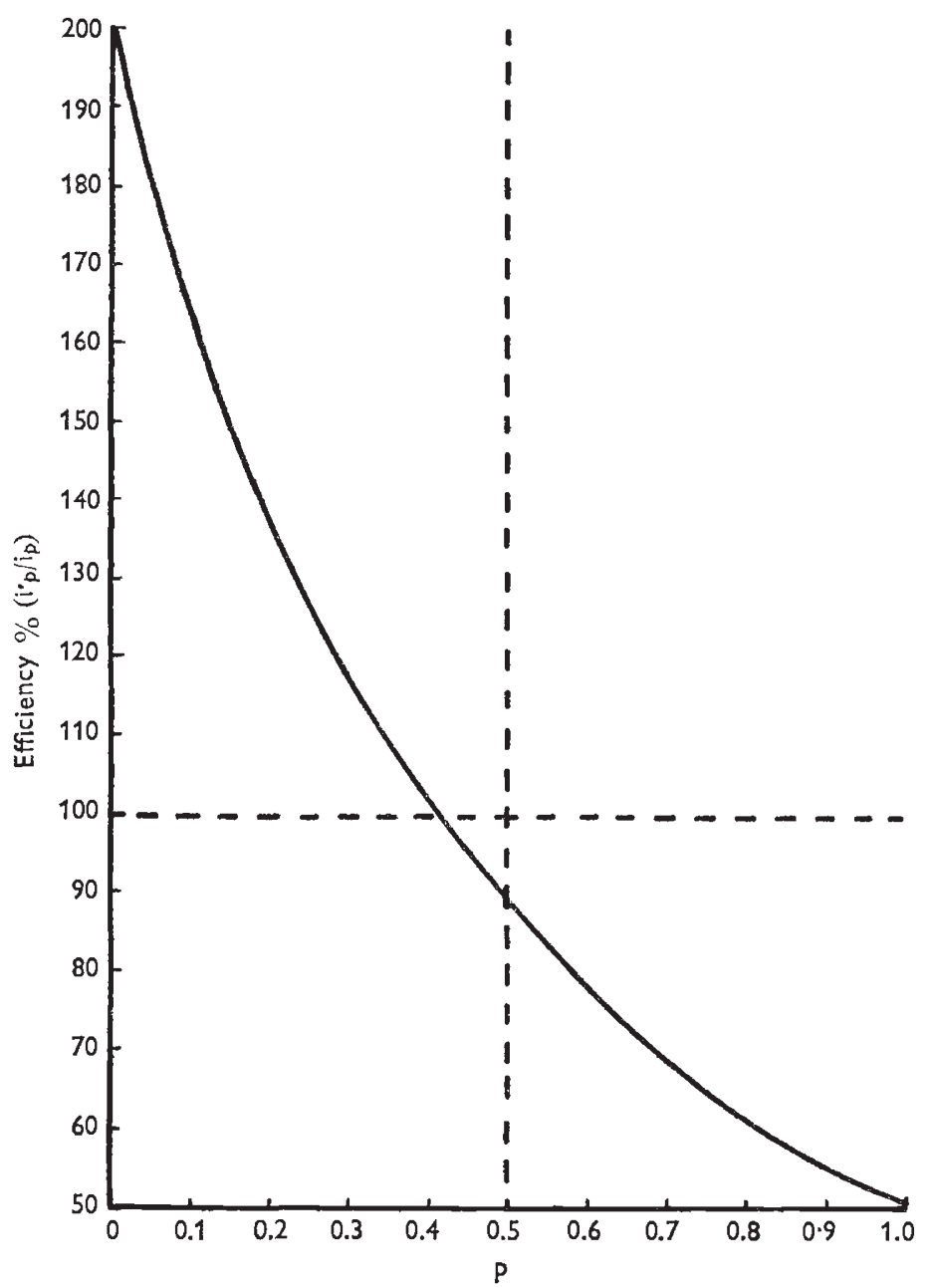

Fig. 1.-The efficiency (\%) of a type 1 A family relative to that of a completely classified intercross (type 1) in respect of the estimation of the male linkage parameter $\mathrm{p}_{\mathrm{m}}$.

gamete of non-recombinant genotype; for example, the cross $A_{1} B_{3} / A_{2} B_{2} \times$ $A_{1} B_{3} / A_{3} B_{1}$ would fulfill this objective. If, however, this cannot be done or the phase of linkage is not known in the parents it is clearly safer to use the type 1 design.

The second point worth making about this design is that it should not be supposed that it is possible to obtain a gain in efficiency by discarding part of the data from a complete family. Thus, suppose that we score $n$ individuals in a type 1 and $n^{\prime}$ individuals in a type IA intercross. 
Then

so that

$$
I_{p_{m}}=\frac{n}{p_{m} q_{m}} \quad \text { and } \quad I_{p_{m}}^{\prime}=\frac{2 n^{\prime}}{\left(1+p_{m}\right)^{2} p_{m} q_{m}}
$$

$$
I_{p_{m}}^{\prime} / I_{p}=\frac{2}{\left(1+p_{m}\right)^{2}} \frac{n^{\prime}}{n}
$$

Supposed now that we discard the $A_{1} B_{1}$ male array from a type 1 family in which it occurs with a frequency of $q_{m} / 2$. Then the total number of individuals in this simulated type $1 \mathrm{~A}$ family is

$$
n^{\prime}=n\left(1-q_{m} / 2\right)=n\left(1+p_{m}\right) / 2
$$

so that

$$
I_{p_{m}}^{\prime} / I_{p_{m}}=\frac{1}{1+p_{m}}
$$

Since $1 /\left(1+p_{m}\right)$ is less than unity for all values of $p_{m}$ greater than zero, it is clearly impossible to obtain a gain in information about $p_{m}$ by discarding the $A_{1} B_{1}$ male array from a complete intercross family.

It should be noted that in theory a similar gain in efficiency could be obtained in a backcross family, though it is difficult to imagine the circumstances in which it would be possible in practice to construct the cross.

\section{The Joint estimate AND Detection OF LINKAge}

When there is no reason to suppose that $p_{f}$ and $p_{m}$ differ in value a joint estimate of $p$ may be obtained. Substituting $p$ for both $p_{f}$ and $p_{m}$ in the $\log$ likelihood expression, and differentiating in respect of $p$ gives as the equation of estimation

$$
\frac{d L}{d p}=\frac{n_{2 .}+n_{3 .}}{p}-\frac{n_{1 .}+n_{4 .}}{q}+\frac{n_{.2}+n_{.3}}{p}-\frac{n_{.4}}{q}-\frac{n}{1+p}=0
$$

in which the first two terms can be recognised as stemming from the female side and the last three from the male. This equation can be reduced to

$$
(\alpha+\beta) p^{2}+(\alpha+3 \beta)-2 \alpha=0
$$

where $\alpha=\left(n_{2 .}+n_{3 .}+n_{.2}+n_{.3}\right)$ and $\beta=\left(n_{1 .}+n_{4 .}+n_{.4}\right)$ with $\alpha+\beta=2 n$. The appropriate root of this quadratic equation provides the joint estimate, $p$.

The amount of information about $p$ is given by the sum of the amounts for $p_{f}$ and $p_{m}$, where they are estimated separately, with, of course, $p$ substituted for $p_{f}$ and $p_{m}$.

Thus

giving

$$
I_{p_{f}}+I_{p_{m}}=n / p_{f} q_{f}+2 n / p_{m} q_{m}\left(1+p_{m}\right)^{2}
$$

$$
I_{p}=\frac{n}{p q}\left[1+\frac{2}{(1+p)^{2}}\right]
$$

The joint expression, $d L / d p$, can also be used to derive the two $\chi^{2}$ 's which provide respectively the joint test of significance for linkage (the 
deviation $\left.\chi_{(1)}^{2}\right)$ and the test of agreement between the male and female sides (the heterogeneity $\chi_{(1)}^{2}$ ). The null hypothesis of no linkage requires that $p=q=\frac{1}{2}$. Substituting these values in $d L / d p$ gives

$$
D=\frac{2}{3}\left[3\left(n_{2 .}+n_{3 .}-n_{1 .}-n_{4 .}\right)+2\left(n_{.2}+n_{.3}-2 n_{.4}\right)\right]=\frac{2}{3}(3 F+2 M)
$$

where $D$ is the departure from the value 0 expected on the null hypothesis - a departure which must arise by sampling variation alone if the null hypothesis is true. The sampling variance of $D$ is $I_{p}$ which when $p=\frac{1}{2}$ is $17 \times 4 n / 9 . \quad D^{2} / I$ is distributed as a $\chi_{(1)}^{2}$ giving us the joint test of significance for linkage

$$
\chi_{(1)}^{2}=\frac{4}{9}(3 F+2 M)^{2} / \frac{17 \times 4 n}{9}=(3 F+2 M)^{2} / 17 n
$$

The test of significance for the heterogeneity between the male and female sides in respect of the evidence they provide for linkage is similarly given by

$$
\chi_{(1)}^{2}=(3 F-2 M)^{2} / 17 n
$$

Adding these two $\chi_{(1)}^{2}$ yields $\chi_{(2)}^{2}=2\left[(3 F)^{2}+(2 M)^{2}\right] / 17 n$, which is not identical with the $\chi_{(2)}^{2}=\frac{F^{2}}{n}+\frac{M^{2}}{2 n}=\frac{2 F^{2}+M^{2}}{2 n}$ obtained by summing the two $\chi^{2}$ 's found on page 124 for the two sides taken separately. This sum would, in fact, correspond to a deviation $\chi_{(1)}^{2}=(\sqrt{ } 2 F+M)^{2} / 4 n$ and a heterogeneity $\chi_{(1)}^{2}=(\sqrt{ } 2 F-M)^{2} / 4 n$.

The difference between this $\chi_{(2)}^{2}$ and that obtained by the maximum likelihood approach is, however,

$$
\begin{aligned}
\frac{1}{2 n}\left(2 F^{2}+M^{2}\right)-\frac{2}{17 n}\left(9 F^{2}+4 M^{2}\right)=\frac{1}{34 n}\left(M^{2}-2 F^{2}\right) & \\
& =\frac{1}{34 n}(M-\sqrt{ } 2 F)(M+\sqrt{ } 2 F)
\end{aligned}
$$

which will vanish when $F=M=0$ (i.e. when the expectations from the null hypothesis are exactly realised) or when $M= \pm \sqrt{ } 2 F$ (i.e. when the weighted deviations of the two sides exactly balance). Thus when the hypothesis of no linkage is acceptable the discrepancy between the $\chi^{2 \text { 's }}$ obtained from the two approaches springs from sampling variation. The maximum likelihood approach is obviously to be preferred but the discrepancy will in any case be small and the alternative $\chi^{2}$ 's can be used without fear of serious misjudgment. If, however, the deviation $\chi^{2}$ is significant, thus rejecting the hypothesis of no linkage, the heterogeneity $\chi^{2}$ found using this hypothesis will inevitably be untrustworthy and could be misleading. In such circumstances heterogeneity should be tested using the value of $p$ estimated from the data, using the method described by Mather (1938, 1951, section 18).

\section{REFERENCES}

LAWRENCE, M. J., CORNISH, M. A., AND HAYWARD, M. D. 1979. Simultaneous estimation of male and female linkage from a single intercross family. Heredity 43, 107-121.

mather, к. 1938, 1951. The Measurement of Linkage in Heredity. Methuen, London. 